\title{
Exact calculation of the trace of the Birman-Schwinger operator of the one-dimensional harmonic oscillator perturbed by an attractive Gaussian potential
}

\author{
S. Fassari ${ }^{1,2,3}$, F. Rinaldi ${ }^{2,3,4}$ \\ ${ }^{1}$ Department of Higher Mathematics, ITMO University, St. Petersburg, Russia \\ ${ }^{2}$ CERFIM, PO Box 1132, CH-6601 Locarno, Switzerland \\ ${ }^{3}$ Dipartimento di Fisica Nucleare, Subnucleare e delle Radiazioni, \\ Univ. degli Studi Guglielmo Marconi,Via Plinio 44, I-00193 Rome, Italy \\ ${ }^{4}$ Istituto Nazionale di Fisica Nucleare, Sezione di Napoli, Naples, Italy \\ silvestro.fassari@uva.es,f.rinaldi@unimarconi.it
}

DOI 10.17586/2220-8054-2019-10-6-608-615

\begin{abstract}
By taking advantage of Wang's results on the scalar product of four eigenfunctions of the 1D harmonic oscillator, we explicitly calculate the trace of the Birman-Schwinger operator of the one-dimensional harmonic oscillator perturbed by a Gaussian potential, showing that it can be written as a ratio of Gamma functions.
\end{abstract}

Keywords: Gaussian potential, Birman-Schwinger operator, trace class operator, harmonic oscillator.

Received: 4 November 2019

\section{Introduction}

As is well known, the harmonic oscillator is one of the very few solvable quantum models, that is to say its eigenfunctions and eigenvalues can be expressed analytically. As a consequence, one of the most fundamental chapters in any quantum mechanics textbook, such as [1], is devoted to its detailed description.

This remarkable property has led theoretical physicists to study various types of models involving perturbations of the harmonic oscillator over many decades. In our inevitably limited summary of the related literature, we have chosen to restrict ourselves to time independent perturbations of the one-dimensional harmonic oscillator.

The numerous contributions spanning many decades can be essentially divided into the following groups:

i) polynomial perturbations;

ii) nonpolynomial perturbations;

iii) point perturbations.

The $x^{4}$-perturbation was the first polynomial perturbation to be extensively investigated also because of its connections with Quantum Field Theory. As the Hamiltonian $-\frac{d^{2}}{d x^{2}}+x^{2}+\beta x^{4}$ is one of the key topics in [2,3], we refer the interested reader to those textbooks and the related references cited therein (see [4] in particular). We remind the reader that the non-analyticity of its eigenvalues as functions of $\beta$ was a well-known thorny issue in the early days of quantum mechanics. However, it is possible to exploit the Borel summability method to get around this obstacle (see Theorems XII.19, XII.21 and Example 1 for both theorems in [3]). A modified Borel transform allows the extension of the method to Hamiltonians of the type $-\frac{d^{2}}{d x^{2}}+x^{2}+\beta x^{2 m}, m \geq 3$ (see Example 3 for Theorems XII.20, XII.21 in [3]). Another noteworthy polynomial perturbation is the one leading to the double well oscillator whose Hamiltonian is given by $-\frac{d^{2}}{d x^{2}}+x^{2}+2 \beta x^{2}+\beta^{2} x^{4}=-\frac{d^{2}}{d x^{2}}+x^{2}(1+\beta x)^{2}$. In particular, the analyticity of its eigenvalues is dealt with in Example 6 following Theorems XII.16.5 in [3] and the related references cited therein. Another important branch of polynomial perturbations is the one of the type $\beta x^{2 m+1}, m \geq 0$, which had its origin in the early investigations on the Hamiltonian of the real cubic oscillator $-\frac{d^{2}}{d x^{2}}+x^{2}+\beta x^{3}$ (see, e.g., [5,6]) and was initially studied in $[7,8]$. Further interest has been drawn into the cubic anharmonic oscillator by the fact that the Hamiltonian $-\frac{d^{2}}{d x^{2}}+x^{2}+i \beta^{\frac{1}{2}} x^{3}, \beta>0$ is $P T$-symmetric (see $\left.[9,10]\right)$.

The most extensively studied nonpolynomial perturbation of the harmonic oscillator is certainly the potential $\frac{\lambda x^{2}}{1+g x^{2}}, \lambda, g>0$ with the early works on this model dating back to the late seventies (see [11-20]). The functional analytic approach based on the Birman-Schwinger principle was used in [21] to analyze the behavior of the eigenvalues for small values of $\lambda$ and large values of $g$. The same technique was exploited in [22] to further investigate this 
model assuming the coupling constant $\lambda$ to be a function of $g$, in particular $\lambda(g)=g^{\frac{n}{2}}, n=1,2,3$. In the last case $\lambda(g)=g^{\frac{3}{2}}$ the Hamiltonian behaves, apart from the constant term $g^{\frac{1}{2}}$, like that of the harmonic oscillator perturbed by a $\delta$-sequence in the range of large values of $g$. More recent contributions to the study of this potential can be found in $[23,24]$.

The one-dimensional harmonic oscillator perturbed by a Dirac distribution centered at the origin was initially considered in [25] as a toy model for the top quark and later in [26] in relation to supersymmetry. It is worth pointing out that the potential $\frac{1}{2} x^{2}-\lambda \delta(x), \lambda>0$ can be regarded as a singular "funnel potential". The same model was subsequently investigated by means of the integral operator isospectral to the Birman-Schwinger operator in [27]. A renewed interest in this model, to a great extent motivated by its applications to Bose-Einstein condensates, has led to more recent contributions such as [28-36]. The case in which the Dirac distribution is not centered at the origin was analyzed in [37] (see also [28]) while the model with two identical deltas symmetrically situated about the origin was thoroughly investigated in [38] (see also [28]). The case of a PT-symmetric Hamiltonian for a onedimensional harmonic oscillator potential decorated with an even number of Dirac delta functions located at symmetric points and complex conjugate strengths was first studied in [39] by means of ODE techniques and later in [40, 41]. As is well known, the Dirac distribution is not the only point interaction in one-dimensional Quantum Mechanics (see [42,43]). Therefore, other types of point perturbations of the harmonic oscillator have been considered more recently, in particular the central $\delta^{\prime}$-potential (also called local $\delta^{\prime}$ in the literature) and the central $\delta^{\prime}$-interaction (also called nonlocal $\delta^{\prime}$ in the literature). The former has been investigated in [44,45] while the latter has been considered in $[46,47]$ (see also [48]). A remarkable property of the latter model is given by the existence of infinitely many level crossings for a critical value of the coupling constant, which leads to a quantum phase transition since the ground state wave function becomes antisymmetric once the coupling constant falls below that critical value.

Although we have restricted our review to point perturbations of the one-dimensional harmonic oscillator, it is worth mentioning that a detailed comparison of the spectral properties of point perturbations of the $n$-dimensional harmonic oscillator, $n=1,2,3$, stressing the role played by the dimension of the underlying space can be found in [50].

In this brief note, we have decided to start investigating the Hamiltonian $H_{-\lambda}=\frac{1}{2}\left(-\frac{d^{2}}{d x^{2}}+x^{2}\right)-\lambda e^{-x^{2}}, \lambda>0$ since the latter Gaussian perturbation has the typical properties of short-range potentials but also those of the harmonic oscillator near the bottom of the well. To the best of our knowledge the only work on this model has been [49]. The combination of the harmonic potential with the attractive Gaussian gives rise to another type of funnel potential which, differently from the aforementioned one or the one analyzed in [51] with a central attractive point perturbation of the conic oscillator, has a finite minimum. The plot shown in Fig. 1 depicts this funnel potential for three different values of the coupling constant. It is rather evident that the Hamiltonian (with a singular funnel potential) $\frac{1}{2}\left(-\frac{d^{2}}{d x^{2}}+x^{2}\right)-\lambda \delta(x)$ is the limit in the norm resolvent sense (see [52]) of the sequence of Hamiltonians (with a finite funnel potential) $\frac{1}{2}\left(-\frac{d^{2}}{d x^{2}}+x^{2}\right)-\lambda n e^{-n^{2} x^{2}}, \lambda>0$ as $n \rightarrow \infty$.

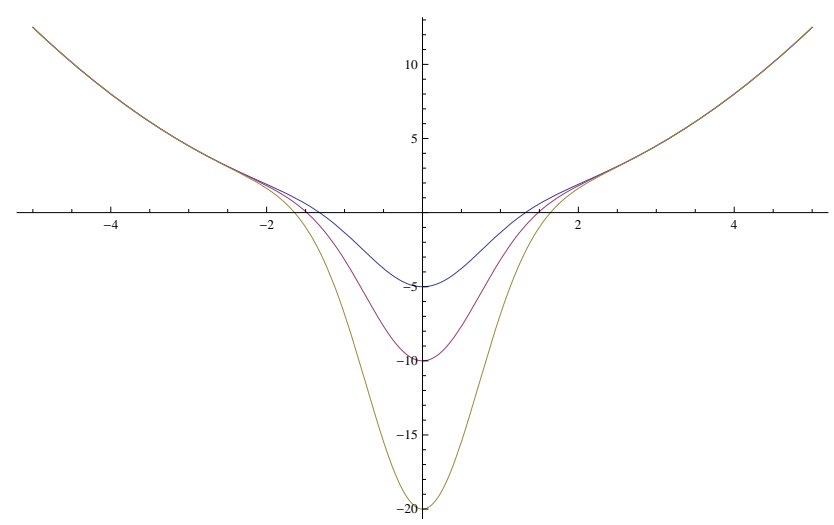

FIG. 1. The plot of $V_{-\lambda}(x)=\frac{1}{2} x^{2}-\lambda e^{-x^{2}}, \lambda>0$ for $\lambda=5,10,20$

Although we are going to focus only on the attractive Gaussian potential $-\lambda e^{-x^{2}}$ in this note, it is clear that the integral operators to be investigated will also be relevant in the case of the repulsive perturbation $\lambda e^{-x^{2}}$ which, 
combined with the harmonic potential, produces the double well potential $V_{+\lambda}(x)=\frac{1}{2} x^{2}+\lambda e^{-x^{2}}, \lambda>0$, depicted in Fig. 2 for three different values of the coupling constant.

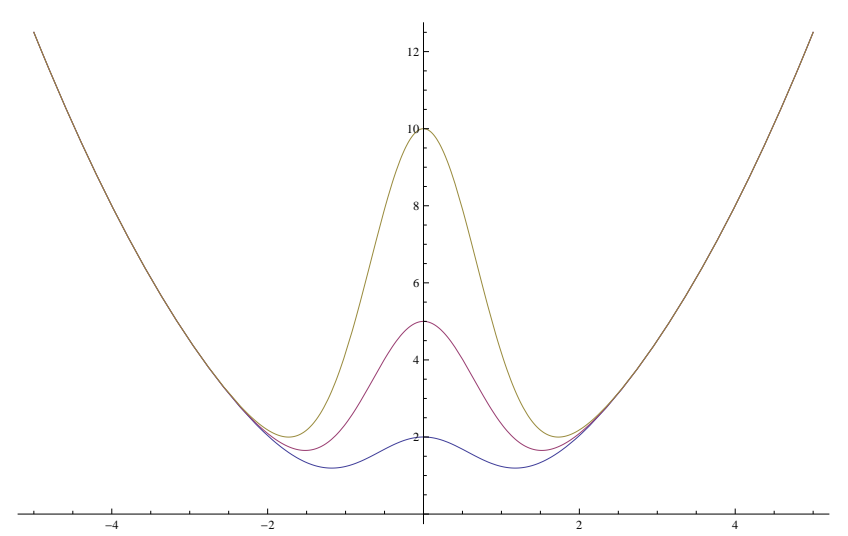

FIG. 2. The plot of $V_{+\lambda}(x)=\frac{1}{2} x^{2}+\lambda e^{-x^{2}}, \lambda>0$ for $\lambda=2,5,10$

In particular, we carry out a detailed analysis of the two isospectral integral operators for this model that are at the heart of the renowned Birman-Schwinger principle, namely $\lambda e^{-x^{2} / 2}\left[H_{0}-E\right]^{-1} e^{-x^{2} / 2}$ and

$\lambda\left[H_{0}-E\right]^{-\frac{1}{2}} e^{-x^{2}}\left[H_{0}-E\right]^{-\frac{1}{2}}$. We show that the trace of these operators can be explicitly written in terms of a ratio of Gamma functions, a feature shared by point perturbations of the harmonic oscillator.

\section{The Birman-Schwinger principle: recasting the Schrödinger equation into an integral equation}

The Schrödinger Hamiltonian for the one-dimensional harmonic oscillator perturbed by an attractive central Gaussian potential reads:

$$
H_{\lambda}=H_{0}-\lambda V(x)=H_{0}-\lambda e^{-x^{2}}, \quad \lambda>0
$$

where

$$
H_{0}=\left(-\frac{1}{2} \frac{d^{2}}{d x^{2}}+\frac{x^{2}}{2}\right) \geq \frac{1}{2}
$$

is the Hamiltonian of the unperturbed harmonic oscillator. Hence, the equation determining the lowest eigenvalue (ground state energy) is given by:

or equivalently,

$$
\left[H_{0}-\lambda e^{-x^{2}}\right] \psi=E \psi, \quad E<\frac{1}{2}
$$

$$
\left[H_{0}-E\right] \psi=\lambda e^{-x^{2}} \psi
$$

By setting $\chi=\left[H_{0}-E\right]^{\frac{1}{2}} \psi$, the latter equation can be rewritten as:

$$
\left[H_{0}-E\right]^{\frac{1}{2}} \chi=\lambda e^{-x^{2}}\left[H_{0}-E\right]^{-\frac{1}{2}} \chi,
$$

which can finally be recast as the following integral equation

$$
\chi=\lambda\left[H_{0}-E\right]^{-\frac{1}{2}} e^{-x^{2}}\left[H_{0}-E\right]^{-\frac{1}{2}} \chi,
$$

taking account that the square root of the resolvent in $x$-space is a positive integral operator for any $E<\frac{1}{2}$.

Alternatively, one can set $\phi=e^{-x^{2} / 2} \psi$, to get instead a different integral equation, namely:

$$
\phi=\lambda e^{-x^{2} / 2}\left[H_{0}-E\right]^{-1} e^{-x^{2} / 2} \phi,
$$


the positive integral operator on the right hand side of the latter equation being the renowned Birman-Schwinger operator widely used in the literature on small perturbations of the Laplacian in the sense of quadratic forms. As is well known (see $[55,56])$, the two integral operators are isospectral.

Therefore, the Birman-Schwinger principle can be exploited in either integral equation to establish the one-to-one correspondence between the ground state energy of the original Hamiltonian and the value of the energy parameter in (2.6) or (2.7) for which the respective integral operator has an eigenvalue equal to one. We wish to remind the reader that the analog of the integral operator in (2.6) for $H_{0}=-\frac{d^{2}}{d x^{2}}$ has been investigated in $[53,54]$ to evaluate the lowest eigenvalues of the one-dimensional negative Laplacian perturbed by an attractive Gaussian potential. It is worth pointing out that in those works the $p$-space representation was chosen so that the square roots of the resolvent acted as multiplication operators and the potential as a convolution integral operator, thus taking advantage of the invariance of the Gaussian under the Fourier integral transform.

\section{Exact calculation of the trace of $\lambda\left[H_{0}-E\right]^{-\frac{1}{2}} e^{-x^{2}}\left[H_{0}-E\right]^{-\frac{1}{2}}$}

As the Gaussian potential is clearly absolutely summable, we could exploit the general estimate of the trace class norm of our positive integral operator given for any absolutely summable perturbing potential in [21] in terms of the $\|\cdot\|_{1}$-norm of the potential, that is to say:

$$
\lambda\left\|\left[H_{0}-E\right]^{-\frac{1}{2}} e^{-x^{2}}\left[H_{0}-E\right]^{-\frac{1}{2}}\right\|_{1}=\lambda \sum_{n=0}^{\infty} \frac{\left(\psi_{n}, e^{-x^{2}} \psi_{n}\right)}{n+\frac{1}{2}-E} \leq \lambda \pi^{\frac{1}{2}} C
$$

where $C=\sum_{n=0}^{\infty} \frac{\left\|\psi_{n}\right\|_{\infty}^{2}}{n+\frac{1}{2}-E}<\infty$, given that the numerator of the sequence inside the sum decays like $n^{-\frac{1}{6}}$ $[27,35,38])$.

It is instructive, however, to take advantage of the unique properties of the Gaussian potential in order to obtain more detailed information on the trace of the operator. Given that $\left(\psi_{n}, e^{-x^{2}} \psi_{n}\right)=\pi^{\frac{1}{2}}\left(\psi_{n}, \psi_{0} \psi_{0} \psi_{n}\right)=$ $\pi^{\frac{1}{2}}\left(\psi_{n} \psi_{0}, \psi_{0} \psi_{n}\right)=\left(\frac{\pi}{2}\right)^{\frac{1}{2}} \psi_{2 n}^{2}(0)$ (having taken account of the fact that the eigenfunctions of the harmonic oscillator are real-valued functions), as follows from Wang's results on such scalar products of four eigenfunctions of the harmonic oscillator (see $[57,58]$ ), one gets:

$$
\lambda \sum_{n=0}^{\infty} \frac{\left(\psi_{n}, e^{-x^{2}} \psi_{n}\right)}{n+\frac{1}{2}-E}=\lambda\left(\frac{\pi}{2}\right)^{\frac{1}{2}} \sum_{n=0}^{\infty} \frac{\psi_{2 n}^{2}(0)}{n+\frac{1}{2}-E}=\lambda(2 \pi)^{\frac{1}{2}} \sum_{n=0}^{\infty} \frac{\psi_{2 n}^{2}(0)}{2 n+1-2 E}
$$

The convergence of the latter series is faster than that of the series defining the constant $C$ in (3.1) since $\psi_{2 n}^{2}(0) \rightarrow$ 0 like $n^{-\frac{1}{2}}$ (see $\left.[27,35,38]\right)$. Furthermore, it is possible to write the series in terms of special functions since:

$$
\lambda(2 \pi)^{\frac{1}{2}} \sum_{n=0}^{\infty} \frac{\psi_{2 n}^{2}(0)}{2 n+1-2 E}=\lambda(2 \pi)^{\frac{1}{2}}\left(H_{0}+\frac{1}{2}-2 E\right)^{-1}(0,0),
$$

the value of the Green function (the integral kernel of the resolvent operator) of the unperturbed harmonic oscillator evaluated at $x=y=0$.

As is well known from the aforementioned papers on point perturbations of the one-dimensional harmonic oscillator $([27,37,38,46])$, the right hand side of $(3.3)$ can be expressed in integral form as:

$$
\lambda(2 \pi)^{\frac{1}{2}}\left(H_{0}+\frac{1}{2}-2 E\right)^{-1}(0,0)=2^{\frac{1}{2}} \lambda \int_{0}^{\infty} \frac{e^{(2 E-1) t}}{\left(1-e^{-2 t}\right)^{\frac{1}{2}}} d t=2^{\frac{1}{2}} \lambda \int_{0}^{1} \frac{s^{-2 E}}{\left(1-s^{2}\right)^{\frac{1}{2}}} d s
$$

As the latter integral can be written in terms of the beta function, which in turn can be expressed as a ratio of Gamma functions, the right hand side of (3.4) is simply given by:

$$
\lambda\left(\frac{\pi}{2}\right)^{\frac{1}{2}} \frac{\Gamma\left(\frac{1}{2}-E\right)}{\Gamma(1-E)}
$$

The result that has just been obtained can be summarized as follows: 
Theorem 3.1. For any $E<\frac{1}{2}$ the positive isospectral integral operators

$$
\left(H_{0}-E\right)^{-1 / 2} e^{-x^{2}}\left(H_{0}-E\right)^{-1 / 2} \text { and } e^{-\frac{x^{2}}{2}}\left(H_{0}-E\right)^{-1} e^{-\frac{x^{2}}{2}}
$$

are trace class and their trace class norm is equal to

$$
\left\|\lambda\left(H_{0}-E\right)^{-1 / 2} e^{-x^{2}}\left(H_{0}-E\right)^{-1 / 2}\right\|_{1}=\lambda\left(\frac{\pi}{2}\right)^{\frac{1}{2}} \frac{\Gamma\left(\frac{1}{2}-E\right)}{\Gamma(1-E)}
$$

Of course, when $E>\frac{1}{2}$ but still in the resolvent set $\rho\left(H_{0}\right)\left(E \neq n+\frac{1}{2}\right)$, the Birman-Schwinger operator is no longer positive so that the right hand side of (3.6) is no longer its $\|\cdot\|_{1}$ - norm but only its trace, that is to say the difference between the norm of its positive part and that of its negative part. The plot of the right hand side of (3.6) is shown in Fig. 3.

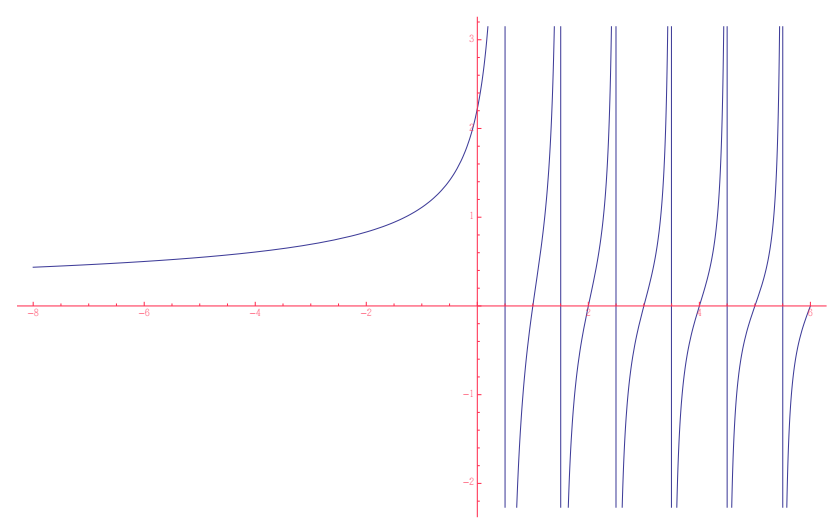

FIG. 3. The plot of the trace of the Birman-Schwinger operator for the Gaussian perturbation as a function of $E$ for $\lambda=1$

As is evident from the graph, the trace is positively (respectively negatively) divergent as $E$ approaches an eigenvalue of $H_{0}$ from the left (resp. right) due to the positive (respectively negative) divergence of the corresponding rank one operator in the left (resp. right) neighbourhood of that eigenvalue.

The reader acquainted with the literature on point perturbations of the one-dimensional harmonic oscillator (see $[25,27,37,38,46])$ will certainly recall that, in the case of an attractive central $\delta$-perturbation, the trace of the positive operator $\lambda\left(H_{0}-E\right)^{-1 / 2} \delta(x)\left(H_{0}-E\right)^{-1 / 2}$ can also be written in terms of a ratio of Gamma functions, namely

$$
\left\|\lambda\left(H_{0}-E\right)^{-1 / 2} \delta(x)\left(H_{0}-E\right)^{-1 / 2}\right\|_{1}=\lambda \frac{\Gamma\left(\frac{1}{4}-\frac{E}{2}\right)}{2 \Gamma\left(\frac{3}{4}-\frac{E}{2}\right)}
$$

The plot of the right hand side of (3.7) is provided in Fig. 4. As was to expected, while the trace corresponding to the Gaussian perturbation vanishes at $E=n, n=1,2, \ldots$ and diverges at $E=n+\frac{1}{2}, n=0,1,2, . .$, the trace corresponding to the $\delta$-perturbation vanishes at $E=2 n+\frac{3}{2}, n=0,1,2, .$. and diverges at $E=2 n+\frac{1}{2}, n=0,1,2, \ldots$

However, a crucial difference between the two models is to be stressed with regard to the quest for the eigenvalues of the perturbed Hamiltonian: while in the latter case, the operator has rank equal to one, which implies that the right hand side of (3.7) determines completely the bound state equation, in the case of the Gaussian perturbation the right hand side of (3.6) represents only the linear term in the expansion of the Fredholm determinant appearing in the bound state equation, that is to say

$$
\operatorname{det}\left[1-\lambda\left(H_{0}-E\right)^{-1 / 2} e^{-x^{2}}\left(H_{0}-E\right)^{-1 / 2}\right]=0,
$$

so that the traces of all the other powers of the operator are needed in order to compute the new eigenvalues created by the perturbation. We remind the reader that, as a consequence of a well-known inequality for Fredholm determinants, for any $\lambda>0$ and $E<\frac{1}{2}$, we get:

$$
\left|\operatorname{det}\left[1-\lambda\left(H_{0}-E\right)^{-1 / 2} e^{-x^{2}}\left(H_{0}-E\right)^{-1 / 2}\right]\right| \leq e^{\lambda \|\left(H_{0}-E\right)^{-1 / 2} e^{-x^{2}}\left(H_{0}-E\right)^{-1 / 2}} \|_{1},
$$




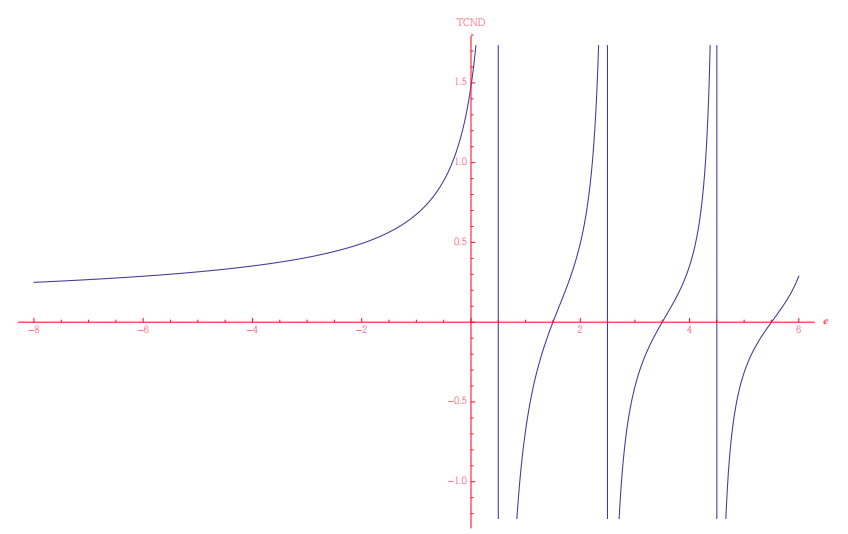

FIG. 4. The plot of the trace of the Birman-Schwinger operator for the $\delta$-perturbation as a function of $E$ for $\lambda=1$.

which becomes

$$
\left|\operatorname{det}\left[1-\lambda\left(H_{0}-E\right)^{-1 / 2} e^{-x^{2}}\left(H_{0}-E\right)^{-1 / 2}\right]\right| \leq e^{\lambda\left(\frac{\pi}{2}\right)^{\frac{1}{2}} \frac{\Gamma\left(\frac{1}{2}-E\right)}{\Gamma(1-E)}},
$$

taking advantage of (3.6). In Fig. 5 the plot of the function on the right hand side of (3.10) is shown for $E<\frac{5}{2}$, even though it must be borne in mind that the function represents the upper bound of the absolute value of the determinant only up to $E<\frac{1}{2}$.

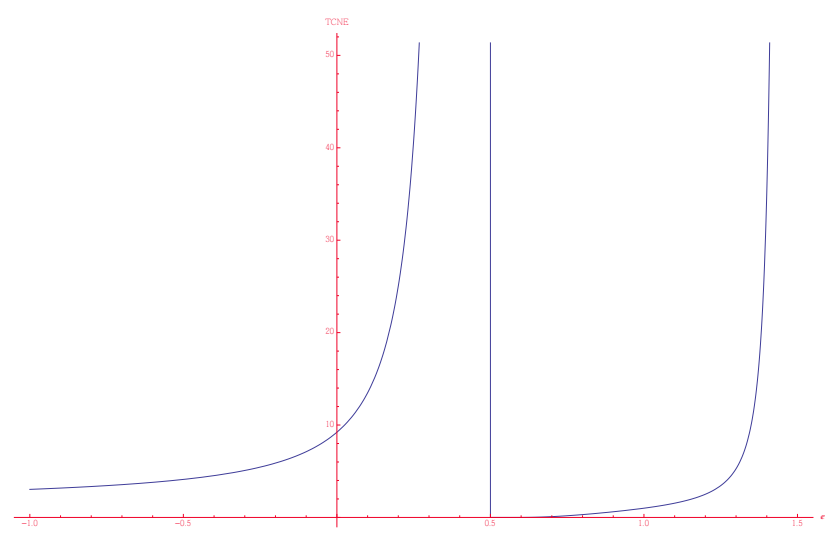

FIG. 5. The plot of $e^{\lambda\left(\frac{\pi}{2}\right)^{\frac{1}{2}} \frac{\Gamma\left(\frac{1}{2}-E\right)}{\Gamma(1-E)}}$ for $\lambda=1$

Despite the far greater complexity of the determinant in our case, it might be possible to compute the eigenvalues of the Hamiltonian of the harmonic oscillator with an attractive Gaussian perturbation to a satisfactory degree of accuracy by exploiting the aforementioned fact that the integral operator $\lambda\left(H_{0}-E\right)^{-1 / 2} e^{-x^{2}}\left(H_{0}-E\right)^{-1 / 2}$ diverges only on a one-dimensional subspace in proximity of an eigenvalue of $H_{0}$, which was crucial in $[21,22]$ to investigate the discrete spectrum of the harmonic oscillator with the Lorentzian perturbation $\frac{\lambda}{1+g x^{2}}$. The latter issue will be dealt with in a separate publication.

\section{Acknowledgements}

The final part of S. Fassari's contribution to this work has been financially supported by the Government of the Russian Federation through the ITMO University Fellowship and Professorship Programme. S. Fassari would like to thank Prof. Igor Yu. Popov and the entire staff at the Departament of Higher Mathematics, ITMO University, St. Petersburg for their warm hospitality throughout his stay. F. Rinaldi wishes to thank Prof. Igor Yu. Popov for inviting him to the Pierre Duclos Workshop over the years as well as for his interest in our research work. 


\section{References}

[1] Landau L.D. and Lifshits E.M., Quantum Mechanics: Non-Relativistic Theory, Pergamon Press.

[2] Reed M., Simon B. Fourier Analysis, Methods in Modern Mathematical Physics, Academic Press, New York, 1975.

[3] Reed M., Simon B. Analysis of Operators, Methods in Modern Mathematical Physics, Academic Press, New York, 1978.

[4] Loeffel J.J., Martin A., Simon B., Wightman A. Padé approximants and the anharmonic oscillator, Phys. Lett. B, 1969,30, P. $656-658$.

[5] Davydov A.S. Quantum Mechanics, Oxford: Pergamon Press, 1965.

[6] Yaris R., Bendler J., Lovett R.A., Bender C.M. and Fedders P.A. Resonance calculations for arbitrary potentials, Phys. Rev. A, $1978,18,1816$.

[7] Caliceti E., Gra S., Maioli M. Perturbation theory of odd anharmonic oscillators, Commun. Math. Phys., 1980, 75 , P. 51.

[8] Caliceti E., Maioli M. Odd anharmonic oscillators and shape resonances Ann. Inst. Henri Poincaré, 1983, XXXVIII(2), P. 175-186.

[9] Fernandez F.M., Guardiola R., Ros J. and Znojil M. Strong-coupling expansions for the $P T$-symmetric oscillators $V(x)=a(i x)+b(i x)^{2}+$ $c(i x)^{3}$. J. Phys. A: Math. Gen., 1998, 31, P. 10105.

[10] Grecchi V., Martinez A. The spectrum of the cubic oscillator. Commun. Math. Phys., 2013, 319, P. 479.

[11] Kaushal S.K. Small $g$ and large $\lambda$ solution of the Schrödinger equation for the interaction $\frac{\lambda x^{2}}{1+g x^{2}}$. J. Phys. A Math. Gen., 1979,12 , P. L253.

[12] Mitra A.K. On the interaction of the type $x^{2}+\frac{\lambda x^{2}}{1+g x^{2}}$. J. Math. Phys., 1979, 19(10), P. 2018-2022.

[13] Bessis N., Bessis G. A note on the Schrödinger equation for the $x^{2}+\frac{\lambda x^{2}}{1+g x^{2}}$ potential. J. Math. Phys., 1980, 21, P. 2780-2785.

[14] Bessis N., Bessis G., Hadinger G. Perturbed harmonic oscillator ladder operators: eigenenergies and eigenfunctions for the $x^{2}+\frac{\lambda x^{2}}{1+g x^{2}}$ interaction. J. Phys. A Math. Gen., 1980, 13, P. 1651.

[15] Bhagwat K.V. A harmonic oscillator perturbed by the potential $\frac{\lambda x^{2}}{1+g x^{2}}$. J. Phys. A: Math. Gen., 1981, 14 , P. 377.

[16] Bessis N., Bessis G. Perturbed factorization of the symmetric-anharmonic-oscillator eigenequation. Phys. Rev A, 1992,46, P. 6824.

[17] Hodgson R.J.W. High-precision calculation of the eigenvalues for the $x^{2}+\frac{\lambda x^{2}}{1+g x^{2}}$ potential. J. Phys. A: Math. Gen., 1988, 21, P. 1563.

[18] Lai C.S., Lin H.E. On the Schrödinger equation for the $x^{2}+\frac{\lambda x^{2}}{1+g x^{2}}$ interaction. J. Phys. A: Math. Gen., 1982, 15, P. 1495.

[19] Blecher M.H., Leach P.G.L. The Schrödinger equation for the $x^{2}+\frac{\lambda x^{2}}{1+g x^{2}}$ interaction. J. Phys. A: Math. Gen., 1987, 20, P. 5923.

[20] Angeletti A., Castagnari C., Zirilli F. Asymptotic eigenvalue degeneracy for a class of one-dimensional Fokker-Planck operators. J. Math. Phys., 1985, 26, P. 678.

[21] Fassari S. A note on the eigenvalues of the Hamiltonian of the harmonic oscillator perturbed by the potential $\frac{\lambda x^{2}}{1+g x^{2}}$. Rep. Math. Phys., 1996, 37(2), P. 283-293.

[22] Fassari S., Inglese G. On the eigenvalues of the Hamiltonian of the harmonic oscillator with the interaction $\frac{\lambda x^{2}}{1+g x^{2}}$. II Rep. Math. Phys., 1997, 39(1), P. 77-86.

[23] Cariñena J.F., Rañada M.F., Santander M. A quantum exactly solvable non-linear oscillator with quasi-harmonic behaviour. Annals of Physics, 2007, 322(2), P. 434-459.

[24] Gadreau P., Safouhi H. Double exponential sinc-collocation method for solving the energy eigenvalues of harmonic oscillators perturbed by a rational function. J. Math. Phys., 2017, 26(10), P. 101509.

[25] Avakian M.P., Pogosyan G.S., Sissakian A.N., Ter-Antonyan V.M. Spectroscopy of a singular linear oscillator. Physics Letters A, 1987, 124(4-5), P. 233-236.

[26] Goldstein J., Lebiedzik C., Robinett R.W. Supersymmetric quantum mechanics: Examples with Dirac $\delta$-functions. American Journal of Physics, 62(7), P. 612-618.

[27] Fassari S., Inglese G. On the spectrum of the harmonic oscillator with a $\delta$-type perturbation. Helv. Phys. Acta, 1994, 67, P. 650-659.

[28] Demiralp E. Bound states of n-dimensional harmonic oscillator decorated with Dirac delta functions. J. Phys. A: Math. Theor., 2005, 22, P. 478393.

[29] Uncu H., Tarhan D., Demiralp E., Müstecaplog̈lu Ö.E. Phys. Rev. A., 2007, 76, P. 013618.

[30] Patil S.H. Harmonic oscillator with a $\delta$-function potential. Eur. J. Phys., 2006, 27, P. 899.

[31] Goold J., ODonoghue D. and Busch Th. Low-density, one-dimensional quantum gases in the presence of a localized attractive potential. $J$. Phys. B: At. Mol. Opt. Phys., 2008, 41, P. 215301.

[32] Shea P., Van Zyl B.P. and Bhaduri R.K. The two-body problem of ultra-cold atoms in a harmonic trap. Am. J. Phys., 2009, 77, P. 5115.

[33] Filatova T.A. and Shafarevich A.I. Semiclassical spectral series of the Schrödinger operator with a delta-potential on a straight line and on a sphere. Theor. Math. Phys., 2010, 164, P. 106480.

[34] Mityagin B. The spectrum of a harmonic oscillator operator perturbed by point interactions. Int. J. Theor. Phys., 2014, 53, P. 118.

[35] Mityagin B.S., Siegl P. Root system of singular perturbations of the harmonic oscillator type operators. Lett. Math. Phys., 2016, 106, P. 147167.

[36] Ferkous N., Boudjedaa T. Bound States Energies of a Harmonic Oscillator Perturbed by Point Interactions. Commun. Theor. Phys., 2017, 67, P 241.

[37] Fassari S., Inglese G. On the spectrum of the harmonic oscillator with a $\delta$-type perturbation. II Helv. Phys. Acta, 1997, 70, P. 858-865.

[38] Fassari S., Rinaldi F. On the spectrum of the Schrödinger Hamiltonian of the one-dimensional harmonic oscillator perturbed by two identical attractive point interactions. Rep. Math. Phys., 2012, 69(3), P. 353-370.

[39] Demiralp E. Properties of a pseudo-Hermitian Hamiltonian for the harmonic oscillator decorated with Dirac delta interactions. Czech. J. Phys., 2005, 55, P. 10814. 
[40] Haag D., Cartarius H. and Wunner G. A Bose-Einstein condensate with $P T$-symmetric double delta function loss and gain in a harmonic trap: a test of rigorous estimates. Acta Polytech., 2014, 54, P. 11621.

[41] Single F., Cartarius H., Wunner G. and Main J. Coupling approach for the realization of a PT-symmetric potential for a Bose-Einstein condensate in a double well. Phys. Rev. A, 2014, 90, P. 042123.

[42] Albeverio S., Dabrowski L. and Kurasov P. Symmetries of Schrödinger operators with point interactions. Lett. Math. Phys., 1998,45 , P. 3347.

[43] Albeverio S. and Kurasov P. Singular Perturbations of Differential Operators: Solvable Type Operators. Cambridge, Cambridge University Press, 2000.

[44] Gadella M., Glasser M.L., Nieto L.M. One-dimensional models with a singular potential of the type $-\alpha \delta+\beta \delta^{\prime}$. Int. J. Theor. Phys., 2011, 50, P. 2144-2152.

[45] Maldonado-Villamizar F.H. Semitransparent one-dimensional potential: a Green's function approach. Phys. Scr., 2015 , 90, P. 065202.

[46] Albeverio S., Fassari S., Rinaldi F. A remarkable spectral feature of the Schrödinger Hamiltonian of the harmonic oscillator perturbed by an attractive $\delta^{\prime}$-interaction centred at the origin: double degeneracy and level crossing. J. Phys. A: Math. Theor, 2013, 46, P. 385305.

[47] Albeverio S., Fassari S., Rinaldi F. The Hamiltonian of the harmonic oscillator with an attractive $\delta^{\prime}$-interaction centred at the origin as approximated by the one with a triple of attractive interactions. J. Phys. A: Math. Theor., 2016, 49, P. 025302.

[48] Cheon T. and Shigehara T. Fermion-Boson duality of one-dimensional quantum particles with generalized contact interactions. Phys. Rev. Lett., 1999, 82, P. 2536.

[49] Nouicer K., Chetouani L. Variational treatment of Gaussian potentials. Acta Physica Slovaca, 1999, 49(3), P. 309-318.

[50] Fassari S., Gadella M., Glasser M.L., Nieto L.M. and Rinaldi F. Level crossings of eigenvalues of the Schrödinger Hamiltonian of the isotropic harmonic oscillator perturbed by a central point interaction in different dimensions. Nanosystems, Physics, Chemistry, Mathematics, 2018, 9(2), P. 179-186.

[51] Fassari S., Gadella M., Glasser M.L., Nieto L.M. Spectroscopy of a one-dimensional V-shaped quantum well with a point impurity. Annals of Physics, 2018, 389, P. 48-62.

[52] Reed M., Simon B. Functional Analysis, Methods in Modern Mathematical Physics. Academic Press, New York, 1972.

[53] Muchatibaya G., Fassari S., Rinaldi F., Mushanyu J. A note on the discrete spectrum of Gaussian wells (I): the ground state energy in one dimension. Adv. Math. Phys., 2016, Article ID 2125769.

[54] Fassari S., Gadella M., Nieto L.M., Rinaldi F. On the spectrum of the 1D Schrödinger Hamiltonian perturbed by an attractive Gaussian potential. Acta Polytech., 2017, 57, P. 385-390.

[55] Klaus M. A remark about weakly coupled one-dimensional Schrödinger operators. Helv. Phys. Acta, 1979, 52, P. 223.

[56] Fassari S. An estimate regarding one-dimensional point interactions. Helv. Phys. Acta, 1995, 68, P. 121-125.

[57] Wang W.-M. Pure Point Spectrum of the Floquet Hamiltonian for the Quantum Harmonic Oscillator Under Time Quasi-Periodic Perturbations. Commun. Math. Phys., 2008, 277, P. 459-496.

[58] Albeverio S., Fassari S., Gadella M., Nieto L.M. and Rinaldi F. The Birman-Schwinger Operator for a Parabolic Quantum Well in a ZeroThickness Layer in the Presence of a Two-Dimensional Attractive Gaussian Impurity. Front. Phys., 2019, 7(102). 